\title{
Real-Time Generative Adaptive Digit Span Testing
}

\author{
John H. Wolfe ${ }^{1}$ \\ San Diego, California
}

This paper explores the subject of generative adaptive testing using the digit span test as an example. A large-sample study of computer-generated and administered digit-span items on Navy recruits showed an almost perfect correlation (.98-.99) between digit span length and IRT difficulty. Predicted IRT parameters can be used for adaptive testing using items generated in real-time. Our results suggest that the best research strategy for developing generative adaptive tests may be to start with the most elementary cognitive tasks, and then build toward more complete psychometric models of complex mental tasks. The results of this study are sufficiently encouraging so that the same research approach should be tried with other forms of memory span tests and more complex working memory tests, including tests for figures, colors, and words The paper advances the conjecture that the test information function of a generative CAT system has a mathematical relationship to the model fit and the distribution of the model-specified item parameters, independent of the content domain of the test.

Keywords: Item generation, Adaptive testing, Digit span, Memory span

Generative testing refers to the automatic production of test items with somewhat predictable difficulty by algorithm or by computer (Bejar, 1993). Generative testing can either be off-line to produce large numbers of alternative candidate items, or in real time, as the examinee takes the test. Real-time generative testing makes cheating impossible. In adaptive testing, the next item is selected or generated based on the current estimate of the examinee's ability inferred from performance on the preceding items of the test. Adaptive testing is more accurate than conventional testing because it reduces measurement error at all levels of ability and the test does not waste time giving items that are too difficult or easy for the examinee. Usually, adaptive testing requires that a bank of items be pre-calibrated for their item response theory (IRT) parameters in advance of operational administration. Real-time generative testing requires that the IRT parameters of each generated item be predictable from the item's features (Embretson,

${ }^{1}$ Corresponding Author: John H. Wolfe, 4310 Hill Street, San Diego, CA 92107, E-mail: wolfe@alumni.caltech.edu

Acknowledgement: The author would like to thank Dr. David L Alderton and Dr. Gerald E. Larson for their assistance in data collection 
1999). One advantage of being able to predict item parameters is that items do not have to be pre-calibrated each time that a new version of a test is developed.

Generative adaptive testing is a methodological advance in testing technology comparable to the development of CAT (Computerized Adaptive Testing) itself. It has several advantages:

1. It promises much greater test security, because an item does not exist until it is generated for a particular examinee.

2. It saves the time of psychometric experts in writing new items periodically.

3. It saves the time and effort needed to calibrate the items in a pool.

4. It forces us to a deeper understanding of how the items in a domain are constructed and what abilities are required to answer them, hopefully leading to greater test validity

The digit span test is one of the simplest of the 11 subtests of the Wechsler Adult Intelligence Scale (Wechsler, 1955). It uses an ad hoc adaptive method in which the examiner orally gives successively longer and longer sequences of digits until the examinee fails two in succession. The digits in a sequence are spoken at the rate of one per second. The final score is an integer representing the length of the largest passed sequence. The scores range from 3 to 9 for forward digit span and 2 to 8 for backward digit span, where the examinee must repeat the digits in reverse order to that in which they were presented. The WAIS-IV added a third component to the total digit span score, Digit Span Sequencing (Lichtenberger \& Kaufman, 2013). I chose to work with the digit span test, not because I think that it is a particularly useful thing to measure, but because it is the simplest and easiest test to generate items from and because there should be a simple relationship between item difficulty and the number of digits in an item.

This paper will show that digit span items can be generated with predictable item parameters, and therefore lend themselves to real-time generative adaptive testing. This paper is a continuation of the work first presented in a draft report of an unfinished project (Wolfe \& Larson, 1990). They found correlations between IRT difficulty and number of digits in a forward digit span test as high as 0.98 . In comparisons of simulated CAT and non-adaptive tests, CAT showed a small increase in reliability, but a substantial increase in test information at the extremes of ability. The present paper presents new results from a different sample, and includes reverse digit span as well as forward digit span.

\section{Method}

\section{Item Generation}

A random number generator was used to generate random digits, which were put in sequences of specified lengths, subject to constraints to reduce the opportunity for simple mnemonic strategies, using the following constraints:

Rule 1: No sequence could begin or end with a zero or one. 
Rule 2: Adjacent digits could not differ by 1.

Rule 3: A sequence could not contain the same digit twice.

\section{Digit Span Tests}

Four "tests" were created off-line consisting of 20 sequences of 5 different lengths ( 5 sets of 4 items each). The two forward digit tests (Forms A and B) used sequences of 5, 6, 7, 8, and 9 digits. The two backward (or reverse) digit tests (Forms A or B) used sequences of 4, 5, 6, 7, and 8 digits. Each test was preceded by a set of six easy practice items of varying lengths.

\section{Subjects}

940 recruits (all male) were tested at the Navy Recruit Training Center in San Diego between August 1990 and September 1991. 907 spoke English as their primary language, 16 spoke Spanish, and 10 Tagalog. There were 45 African-Americans, 32 Asians, 9 Native Americans, and 780 Caucasians in the sample. 192 had some college education and 682 of the remaining examinees were high school graduates. 57 had attended some high school without graduating, and five had eight years or less of education. Their ages ranged from 17 to 34, with a mean of 20.2 , but $90 \%$ were 23 or younger. (Some information was missing on each of the demographic variables.)

\section{Test Administration}

Using IBM PCs, test items were displayed on a 12-inch monochrome monitor. In each item, digits were displayed one at a time for the duration of one second. Examinees entered their responses using the numeric keypad part of the keyboard. Subjects were randomly assigned to take either Form A then B, or B, then A. Within each group, subjects either did either the reverse test first or the forward test, Thus, there were four groups:

1. Forward Form A, Reverse Form A, Forward Form B, Reverse Form B'

2. Forward Form B, Reverse Form B, Forward Form A, Reverse Form A

3. Reverse Form A, Forward Form A, Reverse Form B, Forward Form B

4. Reverse Form B, Forward Form B, Reverse Form B, Forward Form B

\section{Analytical Approach}

Data for forms were combined, regardless of order of administration. Data for Form B were appended to those for Form A. The IRT item parameters for both the one-parameter logistic model and the two-parameter logistic model were computed using the IRTPRO program (Scientific Software International, 2011). Two alternative constraint conditions were employed:

Equality: The items were calibrated under the constraint that items of equal digit length must have the same parameters.

Inequality: The item parameters were determined independently of one another.

\section{Results}

The results for the two-parameter model under equality constraints are displayed graphically in Figure 1, and in tabular form in Table 1. It is not surprising that difficulty should 
increase with the number of digits. It is surprising that the regression should be perfectly linear with such a high correlation.

Table 2 gives the regression equations for the parameters for the various models under equality constraints, and Table 3 gives the regression estimates (i.e. points on the regression line) for those parameters. The later estimates are the best ones that can be derived from the sample data, and that would be applied to newly generated items in real-time adaptive testing ${ }^{2}$. However, they tell little about how individual items might depart from these values. To get an idea of how good the regression of individual items is on number of digits, the analysis was rerun without equality constraints. As can be seen from Figure 2, the predictions of item difficulty are quite good, yielding correlations with digit span of .984 to .991 . The prediction of the discrimination parameter is not so good, dropping to a correlation with number of digits of only .678 .

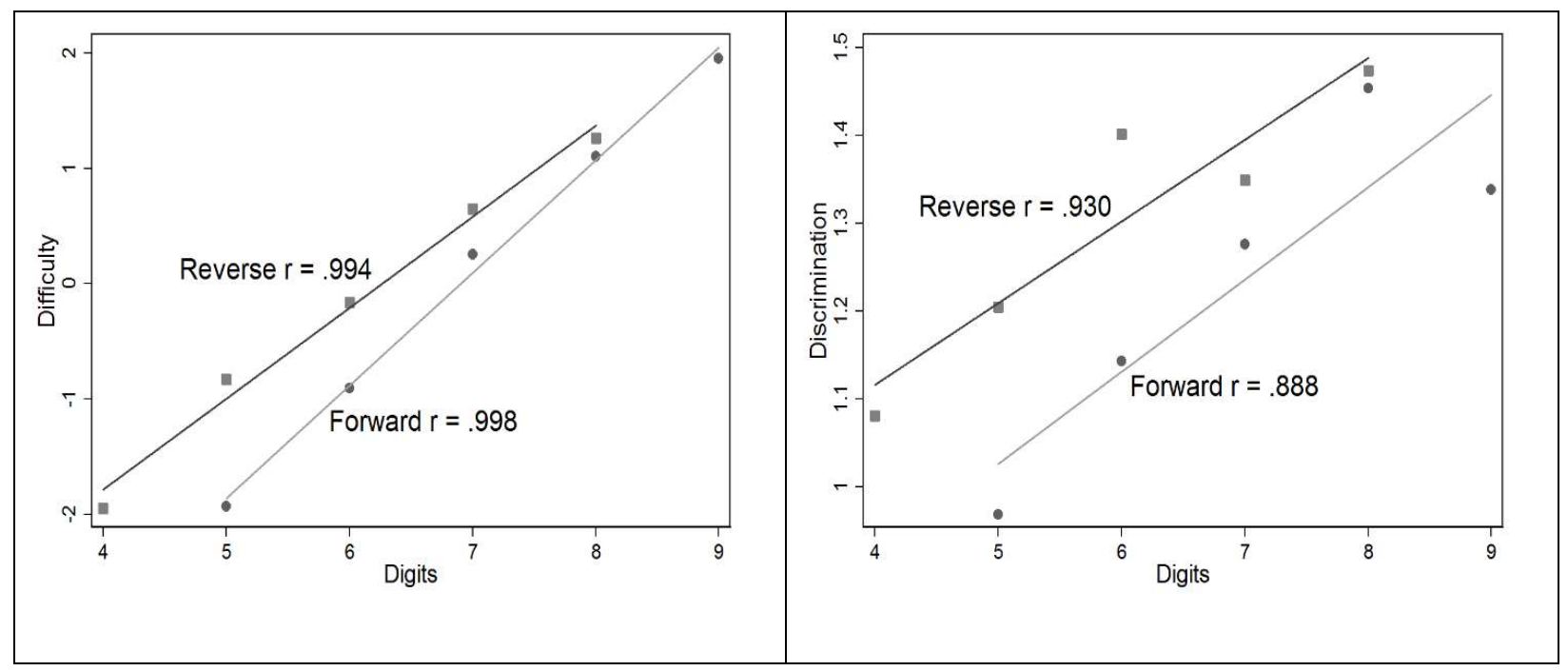

Figure 1. Digit Span IRT Parameters Under Equality Constraints

${ }^{2}$ Variations in display sizes, colors, brightness, and other factors might affect the IRT calibration to an unknown degree, making further experimentation necessary before the parameters derived from this experiment should be used on other equipment. Similar considerations may apply to perceptual and clerical speeded tests. 


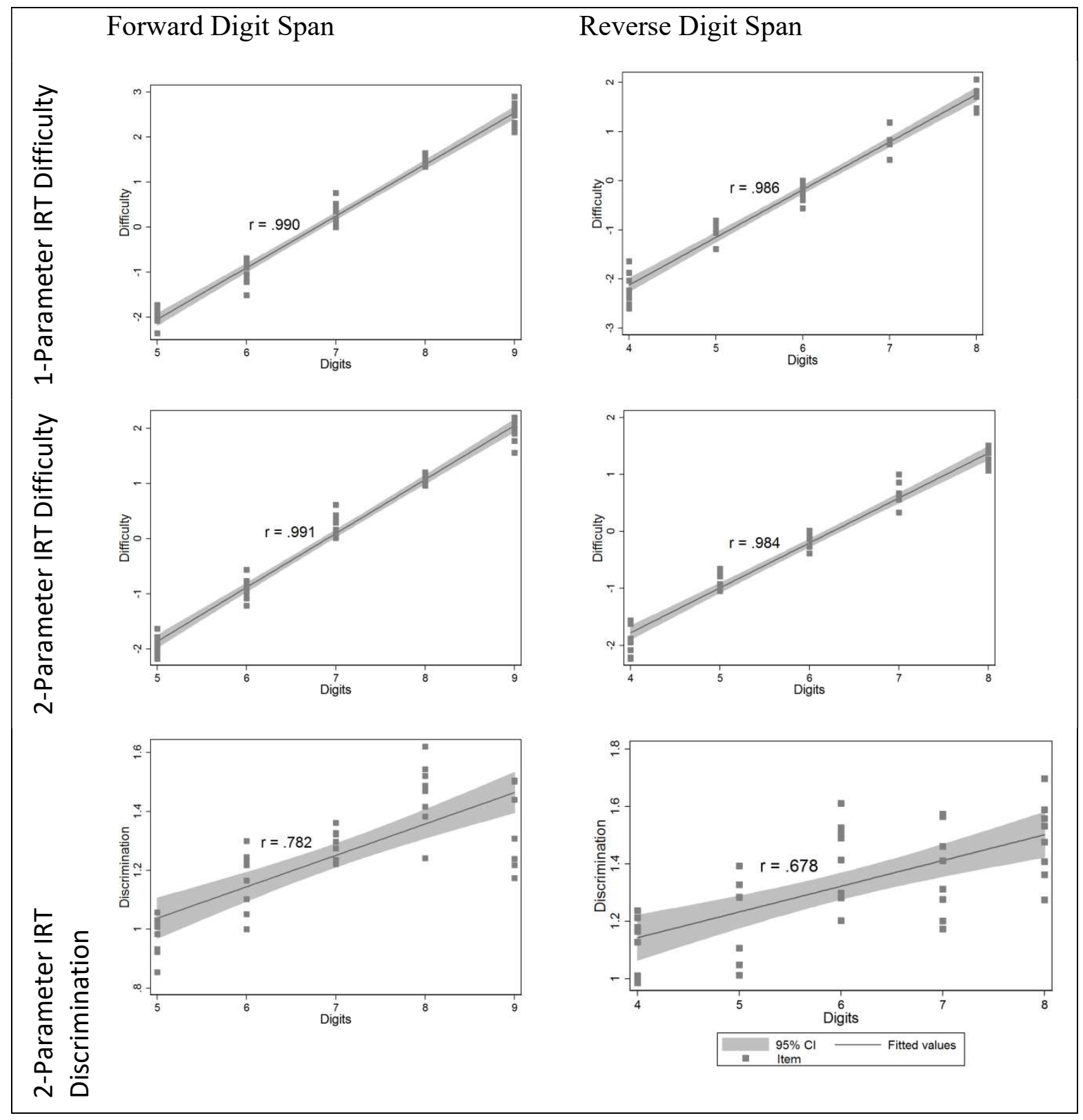

Figure 2. IRT Parameters of Digit Span Items for 1 and 2 Parameter Logistic Models Without Equality Constraints 
Table 1

Model Parameters of Digit Span Items Assuming Equality for Items of Equal Digit Lengths

\begin{tabular}{lllrrrrrr}
\hline Model & Parameter & \multicolumn{1}{c}{ Digits } & 4 & 5 & 6 & 7 & 8 & 9 \\
\hline 1-Parameter & Difficulty & Reverse & -2.1720 & -1.0099 & -0.2370 & 0.8233 & 1.7146 & \\
1-Parameter & Difficulty & Forward & & -1.9653 & -1.0434 & 0.2940 & 1.4508 & 2.4805 \\
2-Parameter & Difficulty & Reverse & -1.9464 & -0.8307 & -0.1639 & 0.6480 & 1.2635 & \\
2-Parameter & Difficulty & Forward & & -1.9313 & -0.9043 & 0.2534 & 1.1034 & 1.9514 \\
2-Parameter & Discrimination & Reverse & 1.0807 & 1.2047 & 1.4013 & 1.3489 & 1.4738 & \\
2-Parameter & Discrimination & Forward & & 0.9683 & 1.1432 & 1.2761 & 1.4535 & 1.3378
\end{tabular}

Table 2.

Regression Coefficients for IRT Parameters with Equality Constraints

\begin{tabular}{lllllll}
\hline Direction & Model & Parameter & Constant & Digits & R & $\begin{array}{c}\text { Root } \\
\text { MSE }\end{array}$ \\
\hline Forward & 1PL & Difficulty & -7.7267 & 1.1386 & 0.9986 & 0.1089 \\
Forward & 2PL & Difficulty & -6.7468 & 0.9773 & 0.9978 & 0.1174 \\
Forward & 2PL & Discrimination & 0.5013 & 0.1049 & 0.8882 & 0.0991 \\
& & & & & & \\
Reverse & 1PL & Difficulty & -5.9401 & 0.9607 & 0.9985 & 0.0963 \\
Reverse & 2PL & Difficulty & -4.9450 & 0.7899 & 0.9943 & 0.1540 \\
Reverse & 2PL & Discrimination & 0.7436 & 0.0930 & 0.9303 & 0.0670 \\
\hline
\end{tabular}

Note: Regression was based on 5 data points 
Table 3.

Regression Estimates of IRT Parameters with Equality Constraints

\begin{tabular}{lllrrrrr}
\hline & & & \multicolumn{5}{c}{ Digits } \\
\hline Direction & Model & Parameter & 5 & 6 & 7 & 8 & 9 \\
Forward & 1PL & Difficulty & -2.0338 & -0.8952 & 0.2433 & 1.3819 & 2.5205 \\
Forward & 2PL & Difficulty & -1.8601 & -0.8828 & 0.0945 & 1.0718 & 2.0491 \\
Forward & 2PL & Discrimination & 1.0259 & 1.1308 & 1.2358 & 1.3407 & 1.4456 \\
& & & 4 & 5 & 6 & 7 & 8 \\
Reverse & 1PL & Difficulty & -2.0975 & -1.1369 & -0.1762 & 0.7845 & 1.7451 \\
Reverse & 2PL & Difficulty & -1.7856 & -0.9958 & -0.2059 & 0.5839 & 1.3738 \\
Reverse & 2PL & Discrimination & 1.1158 & 1.2088 & 1.3019 & 1.3949 & 1.4879 \\
\hline
\end{tabular}

Table 4.

Number of Digits With Highest Information for Ability Range

\begin{tabular}{ccccc}
\hline Direction & \multicolumn{2}{c}{ Forward } & \multicolumn{2}{c}{ Reverse } \\
\hline Model & $1-\mathrm{PL}$ & $2-\mathrm{PL}$ & \multicolumn{1}{c}{ 1-PL } & 2-PL \\
Digits & \multicolumn{2}{c}{ Ability Range } & \multicolumn{2}{c}{ Ability Range } \\
4 & & & $(-2.5,-1.7)$ & $(-2.5,-1.7)$ \\
5 & $(-2.5,-1.5)$ & $(-2.5,-1.7)$ & $(-1.6,-0.7)$ & $(-1.6,-0.9)$ \\
6 & $(-1.4,-0.4)$ & $(-1.6,-0.7)$ & $(-0.6 .0 .3)$ & $(-0.8,0.0)$ \\
7 & $(-0.3,0.8)$ & $(-0.6,0.3)$ & $(0.4,1.2)$ & $(0.1,0.8)$ \\
8 & $(0.9 .1 .9)$ & $(0.4,1.4)$ & $(1.3,2.5)$ & $(0.9 .2 .5)$ \\
9 & $(2.0,2.5)$ & $(1.5 .2 .5)$ & & \\
\hline
\end{tabular}


The results presented so far lend themselves to adaptive administration, which involves estimating the examinee's ability based on the individual's record of item responses and their parameters, and then selecting the next item that gives the highest information for that ability level. For digit span, the second step is easy, since there are only five item lengths to choose from. Table 4 gives a pre-computed guide to item selection, based on information tabulated for each item digit length. To use this table, one (1) decides which model to use, (2) computes the examinee's ability estimate. (3) finds what range the ability falls into, and (4) finds which number of digits to generate at the left-most column of the table. Thus, if the initial estimate of ability is 0.0 , the first item to be administered would have seven digits for forward span and six

Unfortunately, updating the estimates of the examinees' abilities requires computer calculation, and cannot be reduced to table-lookup. It would be desirable to compare the information function of the WAIS sequencing strategy and simple variations of it with that of an optimal sequencing method. Simulations of simplified item sequencing strategies might show that they are almost as good as full ability calculation, but such simulations are beyond the scope of this paper. Also, one should not overlook the advantage of starting with easy items to give the examinee confidence, even if the easy items do not have maximal information.

\section{Discussion}

This paper is a proof of principle that elementary cognitive tasks can have almost perfect predictability of their IRT item parameters, and therefore lend themselves to item generation and adaptive testing. More complex tasks have not been modeled with this degree of fit, probably dues to the presence of multiple alternative mental strategies (MacLeod, Hunt, \& Mathews, 1978) that make the psychometric models incomplete. Several experiments with verbal, quantitative, or figural reasoning items generated by humans following a strict set of rules seldom have been able to predict difficulty with a multiple-R of more than 0.82 (Irvine \& Kyllonen, 2002), (Gierl \& Haladyna, 2013). Our results suggest that the best research strategy for developing generative adaptive tests may be to start with the most elementary cognitive tasks, and then build toward more complete psychometric models of complex mental tasks. For example, the results in this study are encouraging enough so that the same research approach should be tried with other forms of memory span tests and more complex working memory tests, including tests for figures, colors, and words.

Many other researchers have had partial success in generative off-line testing for other domains. Embretson (1999) was able to generate figural reasoning tests, which are likely to be a much better measure of general ability than digit span. Her system predicted item difficulty with a multiple correlation of 0.77 , much lower than the 0.99 obtained with the present study of digit span.

However, multiple correlation in predicting item difficulty may not be the best measure of model fit, because correlation depends on the range (standard deviation) of the difficulties of the items in the study. A better measure might be standard error of item difficulty around the regression line. On the other hand, adaptive testing requires a wide range of item difficulties in order to measure people with very high or low abilities. 
This brings me to another question: how good does the model fit have to be in order to be useful in adaptive testing? Surely, any correlation should be better than a non-adaptive paperand-pencil test. But how does a test based on a given measure of model fit compare with a test based on a pre-calibrated item pool of conventional CAT? Perhaps a multiple R of say, .60, would yield only a little less information than conventional CAT.

I conjecture that the test information function of a generative CAT system has a mathematical relationship to the model fit and the distribution of the model-specified item parameters, independent of the content domain of the test. More research will be required to verify or falsify this hypothesis. 


\section{References}

Bejar, I. C. (1993). A generative approach to psycholgoical and educational measurement. In N. Frederiksen, R. J. Mislevy, \& I. I. Bejar (Eds.), Test theory for a new generation of tests (pp. 323-357). Hillsdale, NJ: Lawrence Erlbaum Associates.

Embretson, S. E. (1999). Generating items during testing: Psychometric issues and models. Psychometrika, 64, 407-433. doi:10.1007/BF02294564

Gierl, M. J., \& Haladyna, T. M. (Eds.). (2013). Automatic item generation: theory and practice. New York: Routledge.

Irvine, S. H., \& Kyllonen, P. C. (Eds.). (2002). Item generation for test development. Mahwah, NJ: Lawrence Erlbaum.

Lichtenberger, E. O., \& Kaufman, A. S. (2013). Essentials of WAIS-IV Assessment (2nd ed.). Hoboken, NJ: John Wiley \& Sons.

MacLeod, C. M., Hunt, E. B., \& Mathews, N. N. (1978). Individual differences in the verification of sentence-picture relationships. Journal of Verbal Learning \& Verbal Behavior, 17, 493-507. doi:http://dx.doi.org/10.1016/S0022-5371(78)90293-1

Scientific Software International. (2011). IRTPRO. Lincolnwood, IL: Scientific Software International. Retrieved from www.ssicentral.com

Wechsler, D. (1955). Manual for the Wechsler adult intelligence scale. New York, New York: The Psychological Corporation.

Wolfe, J. H., \& Larson, G. E. (1990). Generative Adaptive Testing with Digit Span Items. San Diego: Navy Personnel Research and Development Center. https://doi.org/10.31234/osf.io/prs4x 